ARQGA/1191

\title{
ALTERAÇÕES DA MOTILIDADE ESOFAGIANA EM PACIENTES CIRRÓTICOS COM VARIZES DE ESÔFAGO NÃO SUBMETIDOS A TRATAMENTO ENDOSCÓPICO
}

\author{
Priscila Pollo FLORES ${ }^{1}$, Eponina Maria de Oliveira LEMME$^{2}$ e Henrique Sérgio Moraes COELHO ${ }^{1}$
}

RESUMO - Racional - A cirrose hepática apresenta como uma das principais causas de morbimortalidade, a hipertensão porta com o desenvolvimento de varizes esofagianas, possibilidade de hemorragia digestiva alta e agravamento da insuficiência hepática. É importante identificar fatores preditivos causais ou agravantes desta condição e se possível, preveni-los. Nos últimos anos tem se observado a associação de distúrbios motores de esôfago e de refluxo gastroesofágico em pacientes cirróticos com varizes de esôfago. Objetivos - Estudar a prevalência dos distúrbios de motilidade esofagiana e, entre eles, da motilidade esofagiana ineficaz, neste grupo de pacientes e seus possíveis fatores preditivos. Métodos - Avaliaram-se de maneira prospectiva, 74 pacientes com cirrose hepática e varizes esofagianas diagnosticadas por endoscopia digestiva alta, virgens de tratamento endoscópico terapêutico. Todos foram submetidos a um protocolo de investigação clínica, a esofagomanometria e 55 pacientes também realizaram pHmetria esofagiana ambulatorial. Resultados - Alterações da motilidade esofagiana foram observadas em 44 pacientes (60\%), sendo a mais prevalente a motilidade esofagiana ineficaz, verificada em $28 \%$. Refluxo anormal foi encontrado em $35 \%$ dos pacientes. Não houve correlação entre anormalidade manométrica em geral e motilidade esofagiana ineficaz, em particular, e a presença de sintomas esofagianos ou típicos de doença do refluxo, refluxo anormal, a gravidade da doença, a presença de ascite e o calibre das varizes. Conclusões - A maioria dos cirróticos com varizes esofagianas não submetidos a tratamento endoscópico apresenta distúrbios motores do esôfago, sem fatores preditivos identificáveis. A importância clínica desses achados necessita de maior aprofundamento na questão, para elucidar seu papel definitivo.

DESCRITORES - Cirrose hepática. Varizes esofágicas e gástricas. Transtornos da motilidade esofágica.

\section{INTRODUÇ̃̃O E OBJETIVOS}

A cirrose hepática é uma hepatopatia crônica que pode levar a complicações em diversos sistemas do organismo. Entre elas destaca-se a hipertensão porta, que propicia a formação de varizes gastroesofagianas, desenvolvidas por $50 \%$ a $60 \%$ dos pacientes ${ }^{(4)}$. A presença de varizes se correlaciona com o estágio de doença, sendo fonte potencial de sangramento e a principal causa de morte ${ }^{(19)}$. É extremamente importante conhecer os fatores que predispõem ao sangramento e intervir, se possível, buscando redução de mortalidade. Os fatores preditivos já conhecidos são a presença de varizes de grande calibre, a gravidade da hepatopatia e a persistência do consumo de álcool ${ }^{18}$, ${ }^{19)}$, além de fatores locais de diagnóstico exclusivamente endoscópico ${ }^{(4)}$. A presença de esofagite, sobretudo na sua forma erosiva, tem sido implicada no maior risco de sangramento, embora não de forma conclusiva ${ }^{(16)}$. Sabe-se hoje, entretanto, que $50 \%-70 \%$ dos pacientes com doença do refluxo gastroesofágico (DRGE), embora sintomáticos, não apresentam a lesão de mucosa, sendo acometidos de doença do refluxo não-erosiva (DRNE) ou endoscopicamente negativa ${ }^{(12)}$.

A DRGE é uma doença da motilidade em que muitos fatores estão envolvidos, entre eles ressalta-se a existência de distúrbios motores associados, importantes dentro da sua fisiopatologia, uma vez que, ao dificultarem a depuração do ácido refluído, contribuiriam para as lesões da mucosa e complicações da doença ${ }^{(29,33)}$. Entre os distúrbios de motilidade que podem estar associados com DRGE, destacamos a motilidade esofagiana ineficaz, alteração motora mais freqüentemente encontrada e,

Trabalho realizado no Serviço de Gastroenterologia do Hospital Clementino Fraga Filho. Parte de Tese de Mestrado em Gastroenterologia pela Faculdade de Medicina da Universidade Federal do Rio de Janeiro

${ }^{1}$ Serviço de Hepatologia; ${ }^{2}$ Serviço de Gastroenterologia do Hospital Clementino Fraga Filho, Universidade Federal do Rio de Janeiro, Rio de Janeiro, RJ.

Endereço para correspondência: Dra. Eponina M.O. Lemme - Trav. Santa Therezinha, 14 - Tijuca - 20271-070 - Rio de Janeiro, RJ. E-mail: lemme@domain.com.br 
menos comumente, o esôfago em quebra-nozes e o espasmo esofagiano difuso ${ }^{(29)}$.

Os distúrbios motores primários do esôfago, dos quais fazem parte as anormalidades acima referidas, manifestam-se também por disfagia funcional ${ }^{(14)} \mathrm{e}$ dor torácica não-cardíaca ${ }^{(1,31,39)}$, sendo que a motilidade esofagiana ineficaz é um dos principais achados nesses grupos de pacientes.

$\mathrm{Na}$ literatura há poucos estudos que descrevem a motilidade esofagiana em cirróticos com varizes de esôfago não submetidos a tratamento endoscópico ${ }^{(17,45)}$. Entretanto, nenhum deles descreve a prevalência das alterações motoras neste grupo de pacientes e seus possíveis fatores preditivos, importantes na tentativa de se compreender qual o papel destes distúrbios nesta população.

Assim, este trabalho teve como objetivos: avaliar a prevalência dos distúrbios de motilidade esofagiana (DME) e, entre eles, da motilidade esofagiana ineficaz, nos pacientes com cirrose hepática, com varizes esofagianas, não submetidos a terapêutica endoscópica e se estas alterações têm relação com a presença de sintomas, refluxo anormal, existência de ascite, calibre das varizes ou gravidade da cirrose.

\section{PACIENTES E MÉTODOS}

\section{Pacientes}

Este estudo foi aprovada pela Comissão de Investigação Científica do Hospital Universitário Clementino Fraga Filho da Universidade Federal do Rio de Janeiro (HUCFF-UFRJ).

Avaliou-se de maneira prospectiva, 74 pacientes com diagnóstico clínico-laboratorial ou histológico de cirrose hepática, acompanhados no ambulatório de fígado do HUCFF-UFRJ. Todos apresentavam varizes de esôfago, documentadas por endoscopia digestiva alta (EDA) e nenhum havia sido submetido a tratamento endoscópico. Todos os pacientes assinaram o termo de consentimento livre e esclarecido.

\section{Métodos}

Avaliação clínica - Os pacientes foram submetidos a avaliação clínica, que consistiu em preenchimento de um questionário padronizado, contendo dados demográficos, tempo de diagnóstico e etiologia da cirrose. O diagnóstico de cirrose hepática foi baseado na existência das complicações clínicas como ascite, encefalopatia portossistêmica, resultados de métodos complementares (EDA revelando varizes de esôfago e ultra-sonografia abdominal com Doppler, demonstrando hipertensão porta) e em 47 pacientes $(63,5 \%)$ por biopsia hepática. A etiologia da cirrose foi considerada alcóolica, quando a ingesta diária era maior que $80 \mathrm{~g}$ no sexo masculino e $40 \mathrm{~g}$ no sexo feminino ${ }^{(20)}$, pelo vírus $\mathrm{C}$, quando o anti-HVC era positivo, pelo vírus $\mathrm{B}$, quando o HBS-Ag era positivo ${ }^{(41,43)}$, hemocromatose, quando a saturação de transferrina se encontrava acima de $50 \%$, sorologias virais negativas e biopsia hepática com depósitos de ferro pela coloração de Pearls ${ }^{(1)}$, auto-imune, quando os auto-anticorpos (anti-LKM, antimúsculo liso e FAN) eram positivos e sorologias virais negativas, cirrose biliar primária, quando havia anticorpo antimitocôndria positivo e clínica compatível com colestase intra-hepática, cirrose biliar secundária, quando havia evidência demonstrada de obstrução extra-hepática nos métodos de imagem - ultra-sonografia abdominal e/ou tomografia computadorizada. A classificação de Child-Pugh ${ }^{(19)}$ foi utilizada para a avaliação da gravidade da cirrose. $\mathrm{O}$ exame físico foi realizado pelo mesmo observador (PPF), sendo descritos sinais periféricos de insuficiência hepática, quando presentes (eritema palmar, telangiectasias, ginecomastia) e a ascite classificada em: leve, moderada e acentuada ${ }^{(51)}$. A encefalopatia hepática foi considerada presente quando havia flapping, inversão do ciclo sono-vigília, irritabilidade ou alteração de humor, confusão mental e/ou comportamento inadequado ${ }^{(32)}$.

Avaliou-se, também, a presença de sintomas esofagianos, como disfagia (dificuldade no transporte do bolo alimentar até o estômago, ocorrida após o ato da deglutição), dor torácica (dor retrosternal espontânea ou desencadeada por alimentos, em aperto ou queimação, com ou sem irradiação para a região cervical, mandíbula ou dorso) e de sintomas típicos de DRGE, como a pirose (sensação de queimação retrosternal ascendente) e/ou regurgitação (retorno do conteúdo líquido e amargo para a faringe e/ou cavidade bucal).

Exames complementares - Os pacientes foram submetidos a EDA, esofagomanometria e, em 55 deles, foi também realizada a pHmetria esofagiana prolongada.

Endoscopia digestiva alta (EDA) - Os exames foram realizados com o paciente em jejum após anestesia tópica da orofaringe com lidocaína spray a $10 \%$, sob sedação, com diazepam endovenoso em doses variáveis entre 5-10 mg, seguindo a técnica habitual do Setor de Métodos Especiais do Serviço de Gastroenterologia do HUCFF-UFRJ.

As varizes esofagianas (VE) foram classificadas como de pequeno calibre (pequenas e retilíneas - V1), médio calibre (tortuosas, ocupando menos de 1/3 do lúmen - V2) e grosso calibre (tortuosas, ocupando mais de $1 / 3$ do lúmen $-\mathrm{V} 3)^{(6)} \mathrm{e}$ assinalada a presença de varizes gástricas. Gastropatia hipertensiva foi definida pela presença de fino salpico de coloração rosa ou escarlatina e/ou fino padrão reticular branco, separando áreas de mucosa edemaciada e avermelhada (padrão em mosaico) e ou pontos vermelhos difusos ${ }^{(46)}$. Sinais de esofagite, quando presentes, foram descritos conforme a classificação de SAVARYMILLER $^{(49)}$ : grau I - erosões não-confluentes, grau II - erosões confluentes mas não-circunferenciais, grau III - erosões confluentes e circunferenciais, e grau IV - complicações como úlceras, estenose ou esôfago de Barrett. A hérnia de hiato por deslizamento foi definida quando a junção esôfago-gástrica situava-se $2 \mathrm{~cm}$ ou mais acima da impressão diafragmática.

Esofagomanometria (EMN) - Foram empregados dois tipos de equipamentos para a realização dos exames manométricos: 1. Sistema de perfusão, utilizando cateter de polivinil de oito lúmens, diâmetro total de $4,5 \mathrm{~mm}$. As aberturas do cateter são ligadas a transdutores externos de pressão (MX860, MEDEX Inc) e perfundidas durante o exame por uma bomba de perfusão capilar-hidráulica (Biomedics, Califórnia, EUA), a uma velocidade de $0,6 \mathrm{~mL} /$ minuto. As pressões captadas pelos transdutores foram registradas por um polígrafo (PC POLYGRAF VIII, Synectics Medical), convertidas em informações digitais e transferidas para um microcomputador (tipo PC, modelo 486). 2. Sistema com ca- 
teter de transdutores em estado sólido (CTES). As pressões por eles captadas foram registradas em polígrafo SANHILL - EUA e transmitidas de maneira semelhante ao anterior. Os exames foram submetidos a revisão posterior pelo mesmo examinador, empregando-se o software Polygram, versão 4.2 para o sistema de perfusão e Sandhill Bioviews para o sistema de $\operatorname{CTES}^{(9)}$.

O paciente se apresentava para o exame em jejum de 4 horas. Em uma das narinas foi feita anestesia tópica com lidocaína gel, após o que se introduziu o cateter até o estômago. Um sensor de temperatura foi colocado em uma das narinas para registro da respiração, e outro em um dos lados da cartilagem cricóide para registro da deglutição, no caso de emprego do sistema de perfusão.

O exame era, então, iniciado com o estudo do esfíncter inferior do esôfago (EIE). Utilizou-se a técnica de retirada lenta do cateter, com deglutição de 4-6 goles de 3-5 mL de água para estudo dos relaxamentos do EIE. O corpo esofagiano foi estudado com as quatro aberturas proximais do cateter de perfusão ou com os três transdutores em estado sólido, o mais distal de ambos, posicionado $3 \mathrm{~cm}$ acima do limite superior do EIE e feitas 10 deglutições de 3-5 mL de água, com intervalos de pelo menos 20 segundos. Após a realização do exame, foram feitos cálculos executados pelo aplicativo. A pressão de repouso do EIE foi expressa pela média dos quatro valores obtidos em orientação radial, no caso do cateter de perfusão, ou com uma única medida, no caso do CTES, pressões expiratórias máximas. No corpo esofagiano foram avaliadas amplitude, duração, características e velocidade das contrações peristálticas, após as deglutições de água.

Considerou-se normal, baseado nos valores obtidos em 32 voluntários saudáveis estudados no Laboratório de Motilidade de Esôfago do HUCFF-UFRJ ${ }^{(39)}$ :

1. EIE: pressão basal do EIE: $17 \pm 7 \mathrm{~mm} \mathrm{Hg}$, relaxamento completo (resíduo inferior a $8 \mathrm{~mm} \mathrm{Hg}$ ) em pelo menos $80 \%$ das deglutições.

2. Corpo esofagiano: ondas peristálticas em pelo menos $80 \%$ das deglutições de amplitude não inferior a $30 \mathrm{~mm}$ $\mathrm{Hg}$ e com média de amplitude esofagiana distal (ondas peristálticas 3 e $8 \mathrm{~cm}$ acima do EIE) de até $140 \mathrm{~mm} \mathrm{Hg}$ (média +2 DP do valor observado nos voluntários). Contrações simultâneas podem ser encontradas em até $10 \%$ das deglutições.

Critérios utilizados para definição dos distúrbios de motilidade $^{(5,31)}$ :

Acalásia (AC) - relaxamento incompleto do EIE, acompanhado de aperistalse do corpo esofagiano.

Espasmo esofagiano difuso (EED) - contrações simultâneas em 20\% ou mais das deglutições de água, ocupando dois ou três canais de registro consecutivos e distais, intercaladas com peristalse normal. Caso as contrações simultâneas situem-se em dois canais consecutivos, com peristalse acima e abaixo, utiliza-se a denominação de espasmo segmentar.

Esôfago em quebra-nozes $(Q N)$ - as ondas são peristálticas em esôfago distal, mas apresentam amplitude elevada, com média superior a $140 \mathrm{~mm} \mathrm{Hg} \mathrm{(2} \mathrm{DP} \mathrm{acima} \mathrm{da} \mathrm{média} \mathrm{encontrada}$ nos voluntários $\left.{ }^{(38)}\right)$.
EIE hipertenso (EIE hiper) - pressão de repouso do EIE $\geq 32 \mathrm{~mm} \mathrm{Hg}$ (2 DP acima da média encontrada nos voluntários $^{(38)}$, com relaxamento normal).

EIE hipotenso (EIE hipo) - pressão de repouso do EIE $<10 \mathrm{~mm} \mathrm{Hg}$.

Motilidade esofagiana ineficaz (MEI) - ondas de corpo esofagiano distal com diminuição de amplitude $(<30 \mathrm{~mm} \mathrm{Hg})$ ou falha de condução (ausência de onda) em mais de $20 \%$ das deglutições ${ }^{(35)}$.

Distúrbios motores inespecíficos (DMI) - alterações manométricas que não se enquadram nos DME acima citados e que podem vir juntas ou isoladamente. São elas: ondas de triplo pico, ondas retrógradas, ondas de duração aumentada (acima de 6 segundos) e/ou relaxamento incompleto isolado do EIE, em mais de 20\% das deglutições de água.

pHmetria esofagiana prolongada - Utilizou-se registrador portátil digital (MKII ou MKIII Synectics), cateter de antimônio e eletrodo de referência externo. A técnica do exame, descrita em detalhes previamente ${ }^{(36)}$, consiste em anestesia tópica de uma das narinas com lidocaína gel, introdução do cateter e posicionamento $5 \mathrm{~cm}$ acima do EIE, já localizado pela esofagomanometria. $\mathrm{O}$ exame é feito de forma ambulatorial, sendo o paciente instruído a anotar os horários em que se deitou ou se levantou, e os sintomas que porventura ocorressem durante o período de monitorização. Considerou-se refluxo anormal quando a fração de tempo total em que o $\mathrm{pH}$ situou-se abaixo de 4 no período de exame, estivesse acima de 4,5\%(24).

Comparação entre as variáveis categóricas - Os achados de esofagomanometria anormal e do distúrbio motor motilidade esofagiana ineficaz foram comparados com a presença de ascite, calibre das VE, refluxo anormal, sintomas esofagianos, sintomas típicos de refluxo e grau de Child-Pugh da cirrose.

Análise estatística - Foram utilizados o teste Qui-quadrado e teste exato de Fischer. O nível de significância foi de 0,05.

\section{RESULTADOS}

\section{Dados demográficos}

Foram avaliados 74 pacientes, $42(57 \%)$ pertenciam ao sexo masculino e 32 (43\%) ao sexo feminino. A média de idade observada foi de 54 anos, com extremos entre 17 e 85 anos.

\section{Dados clínicos}

A maioria dos pacientes $(44,61 \%)$ apresentava cirrose pelo vírus C. Em seguida, a etiologia alcóolica em 15 (19\%), vírus da hepatite B em $3(4 \%)$ e as demais etiologias agrupadas em 12 (16\%). Em relação à classificação de Child-Pugh, 38 pacientes $(51 \%)$ pertenciam ao grupo A, 29 ao grupo B (39\%) e 7 ao grupo C (10\%). A ascite foi verificada em 35 pacientes (47\%). Destes, $23(65,7 \%)$ apresentavam ascite leve, 5 moderada (14,3\%) e $7(20 \%)$ acentuada.

Quarenta pacientes (54\%) apresentavam pelo menos um sintoma esofagiano e 34 (46\%) eram assintomáticos. Sintomas típicos de DRGE (pirose e/ou regurgitação ácida) foram observados em 32 pacientes (43\%), sendo que pirose ocorreu em $28(38 \%)$ e regurgitação em $18(24 \%)$. Havia dor torácica em 12 (16\%) e disfagia em 5 (7\%), havendo pacientes com superposição de sintomas. 


\section{Métodos complementares}

Endoscopia digestiva alta - em relação ao calibre, as VE foram classificadas como de pequeno calibre (V1), em 46 pacientes (61\%), de médio calibre (V2) em $24(32 \%)$ e grosso calibre (V3) em $5(7 \%)$ pacientes. Havia gastropatia congestiva em 27 (37\%) e varizes gástricas em 5 (7\%).

A esofagite erosiva foi encontrada em três pacientes $(4 \%)$, sendo grau I em dois e grau III de SAVARY-MILLER ${ }^{(49)} \mathrm{em}$ um paciente. Hérnia de hiato foi observada em três pacientes (4\%).

Esofagomanometria (EMN) - a EMN foi anormal em 44 pacientes $(60 \%)$. As alterações motoras encontradas foram: motilidade esofagiana ineficaz: 21 pacientes (28\%) ( 8 com EIE hipo e 1 com EIE hiper); distúrbios motores inespecíficos: 8 pacientes (11\%), todos com relaxamento incompleto do EIE; EIE hipo isolado: 6 pacientes (8\%); esôfago em quebra-nozes: 6 pacientes (8\%); EIE hiper: 2 pacientes (3\%); espasmo esofagiano segmentar: 1 paciente $(1,4 \%)$.

\section{pHmetria esofagiana prolongada}

Realizada em 55 pacientes, demonstrou refluxo anormal em $19(35 \%)$.

\section{Comparação entre as variáveis categóricas}

Ascite $\mathbf{x}$ alteraçóes manométricas - a EMN foi anormal em $21(54 \%)$ dos 39 pacientes sem ascite e em $23(65,7 \%)$ dos 35 com ascite, sem significado estatístico entre essas diferenças $(P=0,42)$. MEI estava presente em $8(20,5 \%)$ dos 39 pacientes sem ascite e em $13(37 \%)$ dos 35 com ascite $(P=0,095)$.

Varizes esofagianas $\mathbf{x}$ alteraçóes manométricas - as Tabelas 1 e 2 demonstram que não houve diferença significativa entre a presença de anormalidade manométrica ou de MEI e o calibre das varizes ( $P=0,99$ e 0,13 , respectivamente).

pHmetria prolongada $\mathbf{x}$ alteraçóes manométricas - a EMN foi anormal em $23(64 \%)$ dos 36 pacientes com $\mathrm{pHmetria}$ normal e em $10(53 \%)$ dos 19 pacientes com pHmetria anormal, não havendo diferença significativa entre o achado de anormalidade manométrica e a presença de refluxo $(P=0,34)$.

MEI estava presente em $10(27,7 \%)$ dos 36 pacientes com pHmetria normal e em 5 (26\%) dos 19 com pHmetria anormal $(P=0,81)$.

TABELA 1 - Calibre das varizes $\mathrm{x}$ esofagomanometria $(\mathrm{n}=74)$

\begin{tabular}{|c|c|c|}
\hline VE & EMN normal & EMN anorma \\
\hline $\mathrm{V} 1(\mathrm{n}=45)$ & $18(40 \%)$ & $27(60 \%)$ \\
\hline $\mathrm{V} 2(\mathrm{n}=24)$ & $10(42 \%)$ & $14(58 \%)$ \\
\hline V3 $(\mathrm{n}=05)$ & $2(40 \%)$ & $3(60 \%)$ \\
\hline Total $(\mathrm{n}=74)$ & 30 & 44 \\
\hline
\end{tabular}

TABELA 2 - Calibre das varizes x motilidade esofagiana ineficaz $(n=74)$

\begin{tabular}{|c|c|c|c|}
\hline \multicolumn{4}{|c|}{ Varizes esofagianas } \\
\hline MEI & V1 & $\mathrm{V} 2$ & V3 \\
\hline Ausente $(\mathrm{n}=53)$ & $29(64 \%)$ & $21(87 \%)$ & $3(60 \%)$ \\
\hline Presente $(n=21)$ & $16(36 \%)$ & $3(13 \%)$ & $2(40 \%)$ \\
\hline Total & $45(100 \%)$ & $24(100 \%)$ & $5(100 \%)$ \\
\hline
\end{tabular}

Grau de Child-Pugh x alteraçóes manométricas - não houve relação entre a gravidade da cirrose e a presença de alterações manométricas ( $P=0,32$ - Tabela 3$)$ ou de MEI ( $P=0,9$ - Tabela 4$)$.

Sintomas esofagianos $\mathbf{x}$ alterações manométricas - a EMN foi anormal em 23 (57\%) dos 40 pacientes com sintomas esofagianos e em 21 (61\%) dos 34 pacientes sem esses sintomas. Sintomas típicos estavam presentes em $17(38,6 \%)$ dos 44 pacientes com manometria anormal e em $15(50 \%)$ dos 30 com EMN normal. Não houve diferença significativa entre o achado de anormalidade manométrica e a presença de sintomas esofagianos ou típicos de refluxo ( $P=0,71$ e $P=0,33$, respectivamente).

TABELA 3 - Esofagomanometria x grau de Child-Pugh $(\mathrm{n}=74)$

\begin{tabular}{|c|c|c|c|c|}
\hline \multirow[b]{2}{*}{ EMN } & \multicolumn{3}{|c|}{ Grau Child-Pugh } & \multirow[t]{2}{*}{ Total } \\
\hline & A & B & $\mathrm{C}$ & \\
\hline Anormal & $22(29 \%)$ & $16(22 \%)$ & $6(8 \%)$ & $44(60 \%)$ \\
\hline Normal & $16(22 \%)$ & $13(17 \%)$ & $1(1 \%)$ & $30(40 \%)$ \\
\hline Total & $38(51 \%)$ & $29(39 \%)$ & $7(10 \%)$ & $74(100 \%)$ \\
\hline
\end{tabular}

TABELA 4 - Motilidade esofagiana ineficaz $\mathrm{x}$ grau de Child-Pugh $(\mathrm{n}=74)$

\begin{tabular}{ccccc}
\hline \multicolumn{4}{c}{ Grau de Child-Pugh } & Total \\
\hline MEI & A & B & C & \\
Ausente & $28(38 \%)$ & $20(27 \%)$ & $5(7 \%)$ & $53(72 \%)$ \\
Presente & $10(13 \%)$ & $9(12 \%)$ & $2(3 \%)$ & $21(28 \%)$ \\
Total & $38(51 \%)$ & $29(39 \%)$ & $7(10 \%)$ & $74(100 \%)$ \\
\hline
\end{tabular}

P-valor: 0,9; MEI - motilidade esofagiana ineficaz

MEI estava presente em $12(30 \%)$ dos 40 pacientes com sintomas esofagianos e em $9(26 \%)$ dos 34 sem esses sintomas $(P=0,74)$. Estava igualmente presente em $9(28 \%)$ dos 32 pacientes com sintomas típicos de refluxo e em 12 (28,5\%) dos 42 pacientes sem esses sintomas $(P=0,97 \%)$

\section{DISCUSSÃO}

É importante que sejam elucidados os fatores envolvidos na morbimortalidade da cirrose hepática, para que seja possível prevenir sua ocorrência. Dentro deste contexto estão a hipertensão porta e as varizes esofagianas, estas com possibilidade de sangramento e todas as suas conseqüências.

A presença ou não do RGE contribuindo para a rotura de VE é assunto controverso. Estudos anatomopatológicos em pacientes que faleceram de HDA por VE demonstraram erosões da mucosa esofagiana em $34 \%-69 \%$ dos casos examinados ${ }^{(15)}$. A avaliação de RGE exclusivamente pela presença de esofagite à EDA, não diagnostica grande parcela de pacientes com DRGE. Cerca de $60 \%$ dos pacientes com DRGE, não apresentam erosões esofagianas ou complicações, sendo denominados de pacientes com doença do refluxo não-erosiva ${ }^{(12)}$.

Há muito tempo é conhecida a associação entre a DRGE e os distúrbios de motilidade. Sabe-se que quanto mais grave a doença, mais intensas as alterações motoras ${ }^{(27)}$. 
O distúrbio de motilidade mais comumente associado à DRGE é a motilidade esofagiana ineficaz, denominação proposta por LEITE et al. ${ }^{(35)} \mathrm{em}$ 1997, para designar a maioria dos pacientes com DMI das classificações anteriores ${ }^{(5)}$ e que passou a fazer parte das mais recentes classificações de DME como distúrbio motor hipocontrátil ${ }^{(52)}$.

Pacientes com cirrose e varizes esofagianas sangrantes submetidos a terapêutica endoscópica, podem apresentar anormalidades motoras esofagianas como conseqüência. Entretanto poucos trabalhos relatam as anormalidades observadas nos pacientes com cirrose e VE sem intervenção endoscópica ${ }^{(16,17,45)}$. Há descrição de redução da amplitude das ondas peristálticas, aumento de sua duração ${ }^{(45)}$ e retardo do tempo de trânsito esofagiano ${ }^{(17)}$, o que poderia, teoricamente, contribuir para a redução da depuração esofagiana de ácido refluído. Isso facilitaria lesão de mucosa como fator precipitante de hemorragia, uma vez que tem sido demonstrado refluxo anormal por pHmetria esofagiana prolongada, em parcela expressiva dessa população ${ }^{(3,42,50)}$. Não se tem conhecimento da prevalência dessas alterações e dos diferentes DME de definição já estabelecida nesse grupo de pacientes. Da mesma forma, se haveria fatores preditivos para a sua existência, daí o motivo da realização deste estudo.

No presente estudo, a EMN foi anormal em 44 dos 74 pacientes $(60 \%)$, sendo a MEI, o DME mais freqüente $(28 \%)$. LEITE et al. ${ }^{(35)}$ demonstraram maior exposição ácida na posição supina e maior tempo de depuração esofagiana à pHmetria prolongada em pacientes com MEI do que o observado em indivíduos normais e aqueles com DME espásticos ${ }^{(10)}$. Correlacionaram, então, a MEI à presença de DRGE. Outros estudos também ligaram a MEI a maior exposição ácida tanto em posição supina, como ereta e o retardo de depuração ácida esofagiana quando comparados com pacientes sem $\operatorname{MEI}^{(2,13,18,22)}$.

A prevalência de MEI é maior em pacientes com DRGE complicada, como o esôfago de Barrett, do que nas formas erosiva e não-erosiva da doença e controles assintomáticos. Nesses últimos foi encontrada prevalência de $10 \%{ }^{(37)}$.

Outros autores, entretanto, contestam a importância da MEI como marcador da DRGE ${ }^{(5)}$. Sugere-se que a MEI poderia se unir às anormalidades funcionais associadas com a DRGE, parece ter seu papel fisiopatogênico, mas poderia não ser seu marcador específico ${ }^{(28)}$.

A MEI é encontrada também em pacientes com outras queixas esofagianas, como disfagia ${ }^{(14)}$ e dor torácica ${ }^{(31,39)}$. Em uma grande série de pacientes com DMI, 95\% dos quais apresentando MEI, a pirose foi a queixa principal, seguida por dor torácica e disfagia ${ }^{(44)}$. Portanto, a MEI é achado comum em pacientes com queixas esofagianas, sendo ou não ligadas à DRGE.

Os distúrbios espásticos (QN, espasmo esofagiano segmentar e EIE-hiper) ${ }^{(10)}$ foram encontrados em nove pacientes $(12 \%)$, sendo o mais freqüente o QN. A prevalência destas anormalidades em portadores de sintomas esofagianos como um todo, é relativamente pequena, porém têm sido observadas em pacientes com dor torácica não-cardíaca e disfagia ${ }^{(14,31,39)}$. O QN tem significado incerto ${ }^{(30)}$, sendo considerado por alguns, como um epifenômeno ou marcador de doença, mais do que sua causa ${ }^{(25,54)}$. Os DMI não-MEI, foram encontrados em sete pacientes $(9,5 \%)$, todos com relaxamento incompleto do EIE. Poder-se-ia especular se a presença mecânica de VE influenciaria o registro do relaxamento do EIE.

Entre os pacientes avaliados no presente estudo, 54\% apresentavam sintomas esofagianos, sendo a pirose o mais comum (38\%), sem correlação com anormalidade manométrica ou com o achado de MEI. Não foi possível a avaliação de cada sintoma em separado, devido ao pequeno número que resultaria de cada variável. O estudo comparativo com relatos de outros trabalhos é difícil, pois não se conhece a prevalência desses sintomas nestes pacientes.

Estudo de 51 pacientes cirróticos com varizes esofagianas demonstrou refluxo anormal à pHmetria prolongada em $37 \%$ dos mesmos. Queixas típicas de refluxo foram preditivas do encontro de pHmetria alterada, levando a se concluir sobre a importância da procura desses sintomas neste grupo de pacientes ${ }^{(50)}$.

No presente estudo a existência de ascite em $47 \%$ dos pacientes, também não esteve relacionada com a presença de alterações manométricas ou de MEI. Aumento de duração de ondas peristálticas e maiores pressões intragástricas foram relatadas em cirróticos com ascite intensa, quando comparados com cirróticos sem ascite ${ }^{(7)}$. Essas anormalidade desapareceram após paracentese, sugerindo que pudessem ser resultado de um componente mecânico ${ }^{(7)}$. A maioria dos pacientes desta série apresentava ascite de grau leve, o que impossibilitou análise estratificada em relação à intensidade da mesma.

Poucos estudos empregaram a pHmetria em pacientes cirróticos com VE. AHMED et al..$^{(3)}$ estudaram por meio de pHmetria, 25 pacientes cirróticos, comparando os resultados aos de 30 indivíduos não-cirróticos, mas com sintomas sugestivos de DRGE. A pHmetria foi anormal em 64\% dos pacientes cirróticos, todos assintomáticos, semelhante aos $70 \%$ de $\mathrm{pHmetria}$ alterada observada no grupo com sintomas sugestivos de DRGE e sem doença hepática. Nenhuma diferença foi encontrada em relação à presença de refluxo e o calibre das varizes.

No presente trabalho havia refluxo anormal em $35 \%$ do 55 pacientes que realizaram a pHmetria. Entretanto, não houve correlação entre o refluxo e as alterações motoras em geral ou MEI, ao contrários dos estudos citados anteriormente ${ }^{(29,35,37,40)}$, sugerindo que nesse grupo de pacientes há outros fatores envolvidos. Esofagite erosiva foi pouco comum (4\%), semelhante ao observado em estudos prévios ${ }^{(3,47)}$, assim como hérnia de hiato (4\%), diferente da população que refere pirose cronicamente, na qual se encontra esofagite em pelo menos $30 \%$ dos pacientes e maior prevalência de hérnia de hiato ${ }^{(33)}$. POLISH et al. ${ }^{(47)}$ demonstraram que, apesar de apenas $9(7,5 \%)$ dos 120 cirróticos com VE estudados apresentarem hérnia hiatal, 8 destes (89\%), cursaram com sangramento varicoso e entre estes últimos, $57 \%$ apresentavam esofagite à EDA. Tais observações implicariam na pesquisa rigorosa de hérnia hiatal nos cirróticos com VE, uma vez que tem sido demonstrada sua importância em retardo do esvaziamento esofágico, aumentando o tempo de contato do ácido com o esôfago ${ }^{(23)}$. A menor prevalência de esofagite erosiva nesses pacientes com cirrose e VE não tem uma justificativa definida. Poder-se-ia interrogar se as varizes de esôfago atuariam como barreira mecânica, em alguma 
fase, embora ocorra a exposição ácida, como demonstrado pela pHmetria. Fatores intrínsecos da mucosa esofagiana na cirrose e hipertensão portal, como mediadores, poderiam contribuir para esse achado.

O estágio da cirrose também não se correlacionou com a presença de DME, o mesmo ocorrendo com o calibre das varizes. A maioria dos pacientes (51\%) foi classificada como Child-Pugh A e também a maioria era de pacientes com $\mathrm{VE}$ de pequeno calibre (61\%). Os pacientes com VE de grosso calibre eram poucos, bem como aqueles em estágio de cirrose Child-Pugh C, devido à dificuldade para a realização voluntária de dois exames invasivos em pacientes freqüentemente descompensados. Não se sabe se a presença de alterações motoras poderia se correlacionar com VE de médio e grosso calibre, caso houvesse maior número de pacientes. Portanto, a análise correlacionando o calibre das varizes, o estágio da cirrose e a presença de ascite pode ter sido prejudicada. Os resultados não foram comparados com o grupo controle de cirróticos sem VE, pela dificuldade na obtenção de tal amostra de pacientes.

Estudo de 1993 revelou amplitude reduzida da peristalse primária quando havia VE de médio e grosso calibre, em relação à observada em voluntários saudáveis ${ }^{(23)}$.

Outro estudo realizado em 1989, também demonstrou motilidade esofagiana alterada, tais como redução da amplitude das contrações na metade inferior do órgão, aumento da duração das ondas e da velocidade da peristalse primária, em cirróticos com VE de médio e grosso calibres. Entretanto, não houve diferenças entre as alterações observadas em pacientes com cirrose sem VE e um grupo controle saudável, sugerindo que as alterações motoras seriam dependentes das varizes e não da cirrose ${ }^{(45)}$. As varizes poderiam exercer efeito mecânico na motilidade do esôfago e na integridade do EIE, resultando em alterações que poderiam influenciar os mecanismos de clareamento esofágico, gerando lesão mucosa e eventual sangramento ${ }^{(17)}$.

Nesta população com amostra significativa de pacientes e a maior em termos de estudo prospectivo sobre o assunto, em que a presença de DME foi importante $(60 \%)$, os sintomas típicos e atípicos freqüentes (54\%), não se demonstrou qualquer fator preditivo para a detecção de DME ou MEI. É possível que alterações mais sutis, verificadas nas médias de amplitude de onda peristáltica, duração e velocidade, comparadas com as encontradas no grupo controle assintomático estudado pelo mesmo laboratório ${ }^{(38)}$, pudessem se tornar aparentes e com fatores preditivos eventuais. Entretanto, não foi objetivo deste estudo, uma vez que se propôs a estudar a prevalência das alterações motoras, já amplamente descritas.

Quais seriam as razões da alta prevalência de DME encontrada no presente estudo, em especial a MEI? Em primeiro lugar, é lícito que se interrogue qual seria a prevalência dessas alterações em indivíduos assintomáticos. Desconhecem-se estudos neste sentido, porém as anormalidades manométricas aqui relatadas são definidas a partir dos valores observados em grupos controles assintomáticos ${ }^{(38,48)}$ e cujas características estão bem estabelecidas na literatura ${ }^{(5,31,54)}$. Não se sabe a real prevalência da MEI em indivíduos assintomáticos, sendo referidos achados compatíveis com este DME em cerca de $10 \%{ }^{(26,37)}$.

Recentemente, o óxido nítrico (ON) assumiu papel importante, como substância vasodilatadora, na exacerbação da hipertensão porta em pacientes com cirrose, e trabalhos têm sido realizados no intuito do maior conhecimento e aplicação clínica desta substância ${ }^{(51)}$. Seu efeito na peristalse esofagiana e EIE começa a ser avaliado. O ON diminui a amplitude da onda peristáltica no esôfago distal e a velocidade da onda peristáltica no esôfago proximal, sendo implicado na etiopatogenia do espasmo difuso ${ }^{(34)}$.

O ON também está envolvido no relaxamento transitório do EIE, fator causal cada vez mais importante na fisiopatologia da DRGE ${ }^{(21)}$.

Na cirrose hepática, como há excesso de $\mathrm{ON}$, este poderia ser o fator causal para o desenvolvimento de refluxo. O refluxo anormal ocorreria, independente de quaisquer fatores associados, como varizes de esôfago e/ou presença de ascite. De forma semelhante, a maior prevalência de DME, principalmente o do tipo MEI, que se caracteriza por redução de amplitude das ondas e falhas de condução, poderia ser relacionada à maior quantidade de $\mathrm{ON}$ existente na circulação dos pacientes cirróticos. Sabe-se que à medida que a cirrose evolui, a vasodilatação aumenta e, mais comumente, ocorre piora da hipertensão porta, as VE aumentam de calibre e a ascite surge ou se agrava. Esta última, pelo aumento da pressão intra-abdominal poderia ter importância no desencadeamento de refluxo, uma vez que já foi demonstrado que a redução da pressão abdominal reduz o refluxo em cirróticos com ascite ${ }^{(42)}$. Todos esses fatores e conseqüências da cirrose, provavelmente, estão interligados numa cadeia de eventos que leva os pacientes à hemorragia digestiva alta e ao óbito.

\section{CONCLUSÕES}

Foi elevada a prevalência de DME, principalmente a MEI, em cirróticos portadores de varizes de esôfago virgens de tratamento. Embora este estudo não tenha trazido a identificação de fatores preditivos de DME nestes pacientes, coloca em perspectiva as implicações potenciais dessas anormalidades, já que foram achado comum nesta população. A associação da MEI com VE necessitaria de estudo em grupo controle sem VE mas, sem dúvida, pode-se identificar nos cirróticos com VE uma população de risco para DME. Todos estes achados reforçam a necessidade de maior esclarecimento sobre a fisiopatologia dos DME e da MEI e sua relevância clínica para os cirróticos.

\section{AGRADECIMENTO}

Os autores agradecem a Ronir Raggio Luiz, Professor Assistente da UFRJ pela cuidadosa análise estatística. 
Flores PP, Lemme EMO, Coelho HSM. Esophageal motor disorders in cirrhotic patients with esophageal varices non-submitted to endoscopic treatment. Arq Gastroenterol 2005;42(4):213-20.

ABSTRACT - Background - The hepatic cirrhosis has as one of the main morbid-mortality causes, the portal hypertension with the development of esophageal varices, the possibility of a digestive hemorrhage and worsening of hepatic insufficiency. It is important to identify causal predictive or aggravating factors and if possible to prevent them. In the last years, it has been observed the association of esophageal motor disorders and gastro-esophageal reflux in cirrhotic patients with esophageal varices. Aims - To study the prevalence of the esophageal motility disorders and among them, the ineffective esophageal motility, in patients with hepatic cirrhosis and esophageal varices, without previous endoscopic therapeutic and the predictives factors. Methods - Prospectively, it has been evaluate 74 patients suffering from liver cirrhosis and esophagic varices, without previous endoscopic treatment. All of them were submitted to a clinical protocol, esophageal manometry and 55 patients also held the ambulatory esophageal pHmetry. Results - Esophageal motility disorders have been found in 44 patients (60\%). The most prevalent was the ineffective esophageal motility, observed in $28 \%$. The abnormal reflux disease was diagnosed through the pHmetry in $35 \%$ of the patients. There were no correlation between the manometrical abnormality in general and the ineffective esophageal motility in particular and the esophageal or gastroesophageal reflux disease symptoms, the abnormal reflux, the disease seriousness, the ascites presence and the gauge of the varices. Conclusions - The majority of cirrhotic patients with non-treated esophageal varices present esophageal motor disorders. No predictive factor was found. The clinical relevance of these findings need more researches in the scope to define the real meaning of theses abnormalities.

HEADINGS - Liver cirrhosis. Esophageal and gastric varices. Esophageal motility disorders.

\section{REFERÊNCIAS BIBLIOGRÁFICAS}

1. Abrahão LJ, Lemme EM. Chest pain of undetermined origin: the role of provocative tests in esophageal investigation [abstract]. Am J Gastroenterol 2002;97(Suppl):S7.

2. Abrahão LJ, Lemme EM, Domingues GR, Carvalho BB Nigri E. Inneffective esophageal motility increases reflux in nonerosive reflux disease patients? [abstract]. Am J Gastroenterol 2002;97(Suppl):s28.

3. Ahmed AM, Al Karawi MA, Shariq S, Mohamed AE. Frequency of gastroesophageal reflux in patients with liver cirrhosis. Hepatogastroenterology 1993;40:478-80

4. Bass NM, Somberg KA. Portal hypertension and gastrointestinal bleeding. In: Mark F, Marvin H, Sleisenger, Bruce F, editors Sleisenger \& Fordtran's gastrointestinal and liver disease; pathophysiology, diagnosis, management. 6th ed. Philadelphia: Saunders, c1998. v. 2, p.1284-309.

5. Benjamin SB, Castell DO. The nutcracker esophagus and the espectrum of esophagea motor disorders. Curr Concepts Gastroenterol 1980;5:3.

6. Beppu K, Inokuchi K, Koyannagi N, Nakayama S, Sakata H, Kitano S, Kobayash M.. Prediction of variceal hemorrhage by esophageal endoscopy. Gastrointest Endosc 1981;27:213-8.

7. Bhatia SJ, Narawane NM, Shalia KK, Mistry FP, Sheth MD, Abraham P, Dherai AJ. Effect of tense ascites on esophageal body motility and lower esophageal sphincter pressure. Indian J Gastroenterol 1999;18:63-5.

8. Boyer TD. Natural history of portal hypertension. Clin Liver Dis 1997;1:31-44

9. Castell JA. Esophageal manometry. In: Castell DO, editor. The esophagus. 2nd ed. Boston: Little, Brown; c1995. p.133-52.

10. Clouse RE, Staiano A. Contractions anormalities of the esophageal body in patients referred for manometry. A new approach to manometric classification. Dig Dis Sci 1983;28:784-91.

11. Conn HO. Portal hypertension, varices, and transjugular intrahepatic portosystemic shunts. Clin Liver Dis 2000;4:133-50.

12. Dent J, Brun J, Fendrick AM and Genval Workshop Group An evidence -based appraisal of reflux disease management- The Genval Workshop Report. Gut 1999;44(suppl 2):s1-16.

13. Diener U, Patti MG, Molena D, Fisichella PM, Way LW. Esophageal dysmotillity and gastroesophageal reflux disease. J Gastrointest Surg 2001;5:260-5.

14. Domingues GRS, Lemme EMO. Diagnóstico diferencial dos distúrbios motores do esôfago pelas características da disfagia. Arq Gastroenterol 2001;38:14-8.

15. Eckardt VF, Grace ND, Kantrowitz PA. Does lower esophageal sphincter incompetency contribute to esophageal variceal bleeding? Gastroenterology 1976;71:185-9.

16. Eckardt VF, Grace ND. gastroesophageal reflux and bleeding esophageal varices. Gastroenterology 1979;76:39-42.

17. Fass R, Landau O, Kovacs TO, Ippolitti AF. Esophageal motility abnormalities in cirrhotic patients before and after endoscopic variceal treatment. Am J Gastroenterol 1997;92:941-6.

18. Fouad YM, Katz PO, Hatlebakk JG. Ineffective esophageal motility: the most commom motility abnormality in patients with GERD-associated respiratory symptoms. Am J Gastroenterol 1999;94:1464-7.
19. Friedman SL. Cirrhosis of the liver and its major sequelae. In: Cecil RL, Bennett JC, Plum F, editors. Cecil textbook of medicine. 20 $0^{\text {th }}$ ed. Philadelphia: Saunders; c1996. p.788-96.

20. Galambos JT. Natural history of alcoholic hepatitis. 3. Histological changes. Gastroenterology 1972;63:1026-35.

21. Hirsch DP, Holloway RH, Tytgat GN, Boeckxstaens GE. Involvement of nitric oxide in human transient lower sphincter relaxations and esophageal primary peristalsis. Gastroenterology 1998;115:374-80.

22. Ho SC, Chang CS, Wu CY, Chen GH, Chun-Ying WU. Ineffective esophageal motility is a primary motility disorder in gastroesophageal reflux disease. Dig Dis Sci 2002;47:652-6.

23. Iwakiri K, Kpbayashi M, Sesoko M, Nomura T. Gastroesophageal reflux and esophageal motility in patients with esophageal varices. Gastroenterol Jpn 1993;28:477-82.

24. Johnson LF, DeMeester TR. Twenty-four-hour $\mathrm{pH}$ monitoring in the distal esophagus. A quantitative measure of gastroesophageal reflux. Am J Gastroenterol 1974;62:325-32.

25. Kahrilas PJ, Dodds WJ, Hogan WJ, Kern M, Amodorfer RC, Reecer A. Esophageal peristaltic dysfunction in peptic esophagitis. Gastroenterology 1986;91:897-904.

26. Kahrilas PJ, Dodds WJ, Hogan WJ. Effect of peristaltic dysfunction on esophageal volume clearance. Gastroenterology 1988;94:73-80.

27. Kahrilas PJ. Nutcracker esophagus: an idea whose time has gone?. Am J Gastroenterol 1993;88:167-9

28. Kahrilas PJ, Pandolfino JE. Ineffective esophageal motility does not equate to GERD. Am J Gastroenterol 2003;98:715-7.

29. Katska DA. Motility abnormalities in gastroesophageal reflux disease. Gastroenterol Clin North Am. 1999;28:905-15.

30. Katz PO, Dalton CB, Richter JE, Wu WC, Castell DO. Esophageal testing of patient with noncardiac chest pain or disphagia. results of three years'experience with 1161 patients. Ann Intern Med 1987;106:593-7.

31. Katz PO, Castell JA. Nonachalasia motility disorders. In: Castell DO, Richter JE, editors. The esophagus. $3^{\text {rd }}$ ed. Philadelphia: Lippincott; c1999. p. 215-32.

32. Kevin D, Mullen D. Hepatic encephalopathy. In: Schiff ER, Sorrell MF, Maddrey WC, editors. Schiff's diseases of the liver. $8^{\text {th }}$ ed. Philadelphia: Lippincott-Raven c1999. v.2, p. $545-83$

33. Klinkemberg-Knol E, Castell DO. Clinical spectrum and diagnosis of gastroesophageal reflux disease. In: Castell DO, Richter JE, editors. The esophagus. $3^{\text {rd }}$ ed. Philadelphia: Lippincott; c1999. p.375-81.

34. Konturek JW, Gullesen A, Domschke W. Diffuse esophageal spasm: a malfunction that involves nitric oxide? . Scand J Gastroenterol 1995;30:1041-5.

35. Leite LP, Johnston BT, Barrett J, Castell JA, Castell DO. Ineffective esophageal motility (IEM): the primary finding in patients with nonspecific esophageal motility disorder. Dig Dis Sci 1997;42:1859-65.

36. Lemme EM, Almeida SM, Firman CM, Nascimento FP. pHmetria esofagiana prolongada - avaliação de 170 exames. Arq Gastroenterol 1997;34:71-7.

37. Lemme EM, Domingues GR, Abrahão-Junior LJ. Gastroesophageal reflux, lowe esophageal sphincter pressure and ineffective esophageal motility in Barrett' s esophagus: a comparative Study. Dis Esophagus 2001;14(Suppl):A093 
38. Lemme EM, Domingues GR, Silva LF, Firman CG, Pantoja JAS. Esofagomanometria computadorizada: resultados preliminares em voluntários adultos saudáveis. GED Gastroenterol End Dig 2001;20:29-35.

39. Lemme EM, Moraes-Filho JP, Domingues GR, Firman CG, Pantoja JA. Manometric findings of esophageal motor disorders in 240 Brazilian patients with non-cardiac chest pain. Dis Esophagus 2000;13:117-21.

40. Lin $\mathrm{S}, \mathrm{Ke} \mathrm{M}, \mathrm{Xu}$ J, Kahrilas PJ. Impaired esophageal emptying in reflux disease. Am J Gastroenterol 1994;89:1003-6.

41. Mello CEB, Mendes CGF, Pernambuco CD. Diagnóstico imunossorológico das hepatites virais. In: Coelho HSM, editor. Gastroenterologia/Hepatites. Rio de Janeiro: Editora Rubio; 2001. p.11-38

42. Navarro-Rodriguez T, Hashimoto CL, Carrilho FJ, Strauss E, Laudanna AA Moraes-Filho JPP. Reduction of abdominal pressure in patients with ascites reduces gastroesophageal reflux. Dis Esophagus 2003;16:77-82.

43. Paixão JBA. Aspectos epidemiológicos das hepatites virais. In: Coelho HSM, editor. Gastroenterologia/Hepatites. Rio de Janeiro: Editora Rubio; 2001. p.1-10.

44. Pantoja JAS. Distúrbios motores inespecíficos em pacientes com sintomas esofagianos [dissertação]. Rio de Janeiro: Universidade Federal do Rio de Janeiro; 1998.

45. Passaretti S, Mazzotti G, Franchis R, Cipolla M, Testoni PA, Tittobello A. Esophageal motility in cirrhotics with and without esophageal varices. Scand J Gastroenterol 1989;24:334-8

46. Payen JL, Calès P, Voigt JJ. Severe portal hypertensive gastropathy and antral vascular ectasia are distinct entities in patients with cirrhosis. Gastroenterology 1995;108:138-44.

47. Polish E, Sullivan BH Jr. Esophagitis associated with hemorrhage from esophageal varices. Ann Intern Med 1961;54:908-11.
48. Richter JE, Wu WC, Johns DN, Blackwell JN, Nelson JL, Castell JA, Castell DO Esophageal manometry in 95 healthy adult volunteers. Variability of pressures with age and frequency of ,abnormal“" contractions. Dig Dis Sci 1987;32:583-92.

49. Savary M, Miller G. The esophagus: handbook and atlas of endoscopy. Solothurn, Switzerland: Verlag Grassmann; 1978. p.135-42.

50. Schechter RB. Prevalência do refluxo gastroesofágico em pacientes cirróticos com varizes de esôfago sem tratamento endoscópico [dissertação]. Rio de Janeiro: Universidade Federal do Rio de Janeiro; 2003.

51. Simpson J A, Conn HO. Role of ascites in gastroesophageal reflux with comments on the pathogenesis of bleeding esophageal varices. Gastroenterology 1968;55:17-25.

52. Spechler SJ, Castell DO. Classification of oesophageal motility abnormalities. Gu 2001;49:145-51.

53. Sogni P, Garnier P, Gadano A, Moreau R, Dall Ava-Santucci J, Danh-Xuan AT, Lebrec D. Endogenous pulmonary nitric oxide production measured from exhaled air is increased in patients with severe cirrhosis. J Hepatol 1995;23:471-3.

54. Vantrappen G, Hellemans J. Oesophageal spasm and other muscular dysfunction. Clin Gastroenterol 1982;11:453-77.

55. Vinjirayer E, Katska DA, Gonzalez B, Brensinger C, Bracy N, Obelmejas R, Metz DC. Ineffective motility is not a marker for gastroesophageal reflux disease. Am J Gastroenterol 2003;98:771-7. 